\title{
Ordered, microphase-separated, noncharged-charged diblock copolymers via the sequential ATRP of styrene and styrenic imidazolium monomers
}

\author{
Zhangxing Shi ${ }^{a}$, Brian. S, Newell ${ }^{b}$,Travis S. Bailey ${ }^{b, *}$, and Douglas L. Gin ${ }^{a, * *}$ \\ aDepartment of Chemistry and Biochemistry, University of Colorado, Boulder, Colorado 80309, USA \\ ${ }^{b}$ Department of Chemical and Biological Engineering, and Department of Chemistry, Colorado State University, \\ Fort Collins, Colorado 80523, USA \\ *Corresponding author: Tel.: +1 970491 4648; E-mail: travis.bailey@ colostate.edu \\ **Corresponding author: Tel.: +1 303492 7640; E-mail: gin@ spot.colorado.edu
}

Keywords: ATRP, imidazolium, poly(ionic liquid), block copolymer, ordered nanostructure

\begin{abstract}
:
A series of imidazolium-based noncharged-charged diblock copolymers (1) was synthesized by the direct, sequential ATRP of styrene and styrenic imidazolium bis(trifluoromethyl)sulfonamide monomers with methyl, $n$-butyl, and $n$-decyl side-chains. Small-angle X-ray scattering studies on initial examples of 1 with a total of 50 repeat units and styrene:imidazolium-styrene repeat unit ratios of $25: 25,20: 30$, and 15:35 showed that their ability to form ordered nanostructures (i.e., sphere and cylinder phases) in their neat states depends on both the block ratio and the length of the alkyl side-chain on the imidazolium monomer. To our knowledge, the synthesis of imidazolium-based BCPs that form ordered, phase-separated nanostructures via direct ATRP of immiscible co-monomers is unprecedented.
\end{abstract}

\section{Introduction}

Polymerized ionic liquids (poly(IL)s) are macromolecules with charged repeat units that are prepared from or structurally related to ionic liquids (ILs). ${ }^{1}$ Since ILs are small-molecule liquid materials with a unique combination of properties (e.g., negligible vapor pressure, ion conductivity, high solubility for certain light gases), ${ }^{2}$ the ability to make processible, solid-state, polymeric analogs is highly desirable. ${ }^{1}$ Poly(IL)s can be made by direct polymerization of IL monomers ${ }^{1}$ with a reactive cation or anion. ${ }^{2}$ They can also be formed by postpolymerization modification of uncharged polymers containing reactive repeat units to generate the charged moieties. ${ }^{1}$ Imidazolium-based poly(IL)s are the most common class of poly(IL)s because of the synthetic versatility, high $\mathrm{CO}_{2}$ gas solubility, and ion conductivity afforded by the imidazolium units. ${ }^{1,2}$ These poly(IL)s have been successfully applied as new membrane materials for $\mathrm{CO}_{2} /$ light gas separations, ${ }^{3}$ solid-state ion conductors, ${ }^{4}$ specialty dispersants/surfactants, ${ }^{5}$ and platforms for new electrochemical devices. ${ }^{6}$

Although imidazolium poly(IL)s have been synthesized in a number of polymer chain architectures ${ }^{1}$ one motif that has received a great deal of recent research attention is phaseseparated, poly(IL)-based block copolymers (BCPs). BCPs can form phase-separated, 
nanostructures ranging from simple micelles and vesicles to periodic assemblies with 1D, 2D, or 3D order, making them attractive for encapsulation/release and transport applications. ${ }^{7}$ In addition, alteration of BCP chemical composition and block lengths can be used to obtain different mechanical properties and control the phase-separated morphologies formed. ${ }^{7}$ If one of these blocks contains imidazolium salt units, then nanostructured BCPs can be generated with one domain having imidazolium IL-like properties, resulting in a material with features of BCPs and poly(IL)s.

Over the past few years, several types of imidazolium-based BCPs containing charged and uncharged blocks have been prepared using three general approaches: (1) sequential controlled/living polymerization of an imidazolium-based monomer with an uncharged comonomer; (2) controlled/living polymerization of an imidazolium monomer from the end of an uncharged polymer; or (3) postpolymerization functionalization of an uncharged BCP containing a reactive segment to generate imidazolium units in situ. The first approach has included reversible addition-fragmentation chain-transfer (RAFT) copolymerization of acrylate-, acrylamide-, or vinyl-substituted imidazolium monomers with conventional acrylates and acrylamides to give diblock noncharged-charged copolymers; ${ }^{8-10}$ and sequential ring-opening metathesis polymerization (ROMP) of alkyl ester- and imidazolium-containing norbornene monomers. ${ }^{11}$ It should be noted that shortly after this manuscript was submitted for publication, a report on the sequential atom-transfer radical polymerization (ATRP) of an imidazolium-styrene monomer and styrene appeared on-line. ${ }^{12}$ However, the resulting BCPs did not form phaseseparated nanostructures and were characterized only for their molecular weights. ${ }^{12}$ The second approach has utilized ATRP ${ }^{13,14}$ and RAFT $^{15,16}$ of acrylate- ${ }^{13,15,16}$ and styrene-based ${ }^{14}$ imidazolium monomers to form poly(IL) segments off the end(s) of uncharged telechelic ${ }^{13-15}$ or monofunctional ${ }^{16}$ polymers. The third approach has involved substitution of imidazole onto uncharged BCPs containing a reactive alkyl halide block made by nitroxide-mediated polymerization (NMP), ${ }^{17-19}$ RAFT, ${ }^{20}$ or step-growth $^{21,22}$ polymerizations; selective quaternization of polystyrene- $b$-poly(styrene-imidazole)s formed by NMP; ${ }^{23}$ or attachment of imidazolium groups onto uncharged thiol-acrylate-based BCPs made by RAFT. ${ }^{24}$ One other related polymer modification approach is cation-exchange of commercial alkyl-anionic BCPs with imidazolium salts to generate BCPs with free imidazolium counterions. ${ }^{25}$ Although many of these materials have been demonstrated to be useful in several applications, ${ }^{11 \mathrm{c}, 11 \mathrm{~d}, 16,18,20-25}$ only a small subset of them has been reported to form phase-separated, ordered nanostructures in the solvent-free melt state. ${ }^{11,18,21 \mathrm{c}, 22,23 \mathrm{~b}, 24}$ The majority of the BCPs listed above were not reported to form periodic nanostructures, ${ }^{12-15,20}$ or they only formed micelles or non-ordered phases. $^{8-}$ $10,16,17,19,21 \mathrm{a}, 21 \mathrm{~b}, 23 \mathrm{a}, 25$

In 2011, our research team was one of the first to show that imidazolium-based BCPs can form ordered nanostructures in their neat state by using alkyl-imidazolium BCPs made from sequential ROMP of a norbornene-based alkyl ester monomer and a norbornene-based imidazolium monomer. ${ }^{11 a}$ Supported membranes of these materials were also found to have nanostructure-dependent $\mathrm{CO}_{2}$ transport properties, making them potential useful for gas separations. ${ }^{11 c, d}$ However, there were several limitations with our initial IL-based BCP design that hampered preparation of substantial amounts of these materials for exploring applications (e.g., the use of an expensive Ru-based ROMP catalyst, residual metal contamination in the BCPs (from the catalyst) after isolation, and elaborate monomer syntheses). ${ }^{11}$ 
Herein, we show imidazolium-based noncharged-charged diblock copolymers (1) that form ordered, phase-separated nanostructures in their neat melt states can be synthesized by the sequential ATRP of styrene and several styrenic imidazolium bis(trifluoromethyl)sulfonamide $\left(\mathrm{Tf}_{2} \mathrm{~N}^{-}\right)$monomers with different length alkyl side-chains (2a-c) (Fig. 1). Studies on nine initial examples of 1 with a total of 50 repeat units and styrene:imidazolium-styrene repeat unit ratios of 25:25, 20:30, and 15:35 indicated that their ability to form ordered nanostructures depends on both the block ratio and the length of the alkyl chain on the imidazolium-styrene monomer. Compared to our previous ROMP method, this controlled radical polymerization (CRP) approach with styrenic monomers allows the use of a less expensive controlled/living polymerization catalyst, minimum metal contamination in the BCPs formed, and more facile and scalable monomer syntheses. Although ATRP homopolymerization of imidazolium-containing monomers is well-established, ${ }^{1}$ to our knowledge the direct, sequential ATRP of imidazolium and hydrophobic monomers to generate imidazolium BCPs with ordered, phase-separated nanostructures has not been reported. ${ }^{8-10,12}$ As noted previously, the synthesis of poly(imidazolium-styrene)- $b$-polystyrene BCPs via ATRP that was reported shortly after this manuscript was submitted did not generate BCPs with ordered, phase-separated nanostructures. ${ }^{12}$ That work also utilized a different type of ATRP (i.e., ARGET ATRP), employed a different monomer polymerization sequence (i.e., imidazolium-styrene then styrene), and polymerized just one imidazolium-styrene monomer (2b) instead of a range of imidazolium monomers with different side-chain lengths. ${ }^{12}$

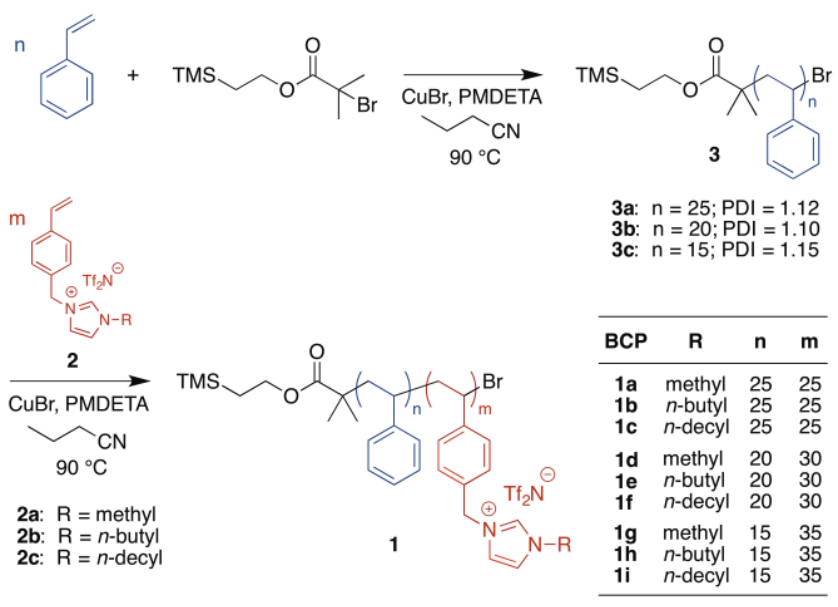

Fig. 1. Synthesis and structures of the noncharged-charged BCPs made via sequential ATRP in this study.

\section{Results and discussion}

\subsection{Materials synthesis}

As a more economical and scalable alternative to ROMP, CRP was selected because of its molecular weight (MW) and block ratio control, low PDI, and ability to polymerize a broad range of acrylate and styrene monomers (including ionic versions). Compared to the other CRP 
methods (NMP, RAFT), ATRP was chosen because it utilizes inexpensive and commercially available catalysts and ligands. Also, our initial studies showed that ATRP was more promising for the controlled polymerization of the styrenic imidazolium monomers in terms of conversion and reaction time (see the Experimental Section).

Styrenic compounds are ideal monomers for ATRP-based BCP synthesis because of their facile synthesis and functional group attachment. Monomers $\mathbf{2 a}$ and $\mathbf{2 b}$ were prepared by reacting 4-vinylbenzyl chloride with the appropriate $N$-alkylimidazole, followed by ion-exchange of the $\mathrm{Cl}^{-}$anion with $\mathrm{Tf}_{2} \mathrm{~N}^{-}$according to literature procedures. ${ }^{26,27 a}$ Compound $2 \mathrm{c}$ is a new monomer and was synthesized using similar methods (see the Experimental Section).

As shown in Fig. 1, BCPs 1a-i were synthesized via ATRP of first styrene and then monomers $\mathbf{2 a}$, 2b, or 2c using $\mathrm{CuBr} / N, N, N^{\prime}, N^{\prime}, N^{\prime \prime}$-pentamethyldiethylenetriamine (PMDETA) as the catalyst system and 2-(trimethylsilyl)ethyl 2-bromo-2-methyl-propanoate as the initiator in butyronitrile solution (see the Experimental Section and Supplementary Data for details). Analysis of the isolated BCPs formed by this method show very little if any residual $\mathrm{Cu}$ from the polymerization catalyst (see the Supplementary Data). In our sequential copolymerizations, controlled-length, low-PDI reactive polystyrene blocks 3a-c were first synthesized and then used as ATRP macro-initiators to form the subsequent attached imidazolium block upon addition of the appropriate amount of $\mathbf{2 a}, \mathbf{2 b}$, or $\mathbf{2 c}$. This polymerization sequence was important because of the lower efficiency of the initiator system with the imidazolium-styrene monomers and the inconvenient purification process of the resulting ionic macro-initiator if the imidazolium block were formed first.

\subsection{Confirmation of controlled polymerization behavior for the ATRP of styrenic imidazolium monomers $2 a-c$}

At the start of this work, the ATRP, ${ }^{27} \mathrm{NMP},{ }^{28}$ and $\mathrm{RAFT}^{27 \mathrm{~b}}$ of only two styrenic imidazolium monomers (2b and a chiral cyclohexanol-substituted imidazolium-styrene monomer $^{27 f}$ ) were reported in the literature. The controlled ATRP of $\mathbf{2 a}$ and $\mathbf{2 c}$ had not been reported, and the sequential ATRP of styrene and $\mathbf{2 a - c}$ to generate nanostructured BCPs was also unprecedented. In order to confirm the controlled ATRP of $\mathbf{2 a}$ and $\mathbf{2 c}$ for viable BCP synthesis with styrene, the homopolymerization kinetics of $\mathbf{2 a}$ and $\mathbf{2 c}$ were first monitored. In addition, the kinetics of the copolymerizations of styrene with $\mathbf{2 a - c}$ were also studied to confirm controlled polymerization behavior. In these initial studies, monomer consumption and the absolute number-average MW $\left(M_{\mathrm{n}}\right)$ values of the formed polymers were calculated by ${ }^{1} \mathrm{H}$ NMR integration and end-group analysis (see the Supplementary Data). As can be seen in Fig. 2, the ATRP of $\mathbf{2 a}$, the ATRP of $\mathbf{2 c}$, and the sequential ATRP of styrene followed by $\mathbf{2 a}$ (as a representative copolymerization example) all show linear, first-order monomer conversions with time and linear increases in $M_{\mathrm{n}}$ with monomer conversion. This behavior indicates that these ATRP homopolymerizations and copolymerizations are well-controlled. By varying the styrene and 2a-c mole ratios in the sequential ATRP reactions, nine BCPs (1a-i) were obtained with three different styrene:imidazolium-styrene block ratios and three different alkyl side-chain lengths on the imidazolium-containing blocks. 

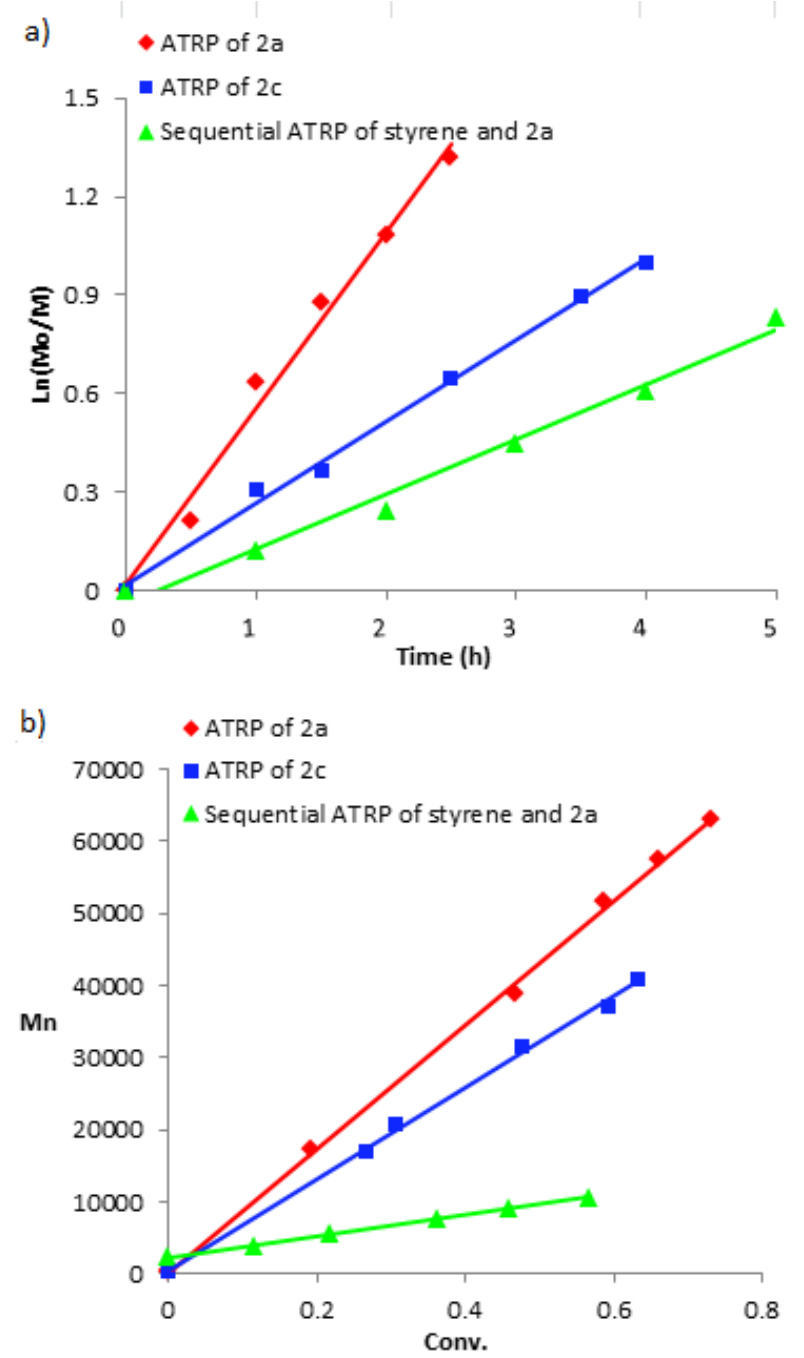

Fig. 2. Plots confirming the controlled ATRP homopolymerization of monomers $\mathbf{2 a}$ and $\mathbf{2 c}$, and the sequential ATRP of styrene and 2a to form BCP $\mathbf{1 d}$.

\subsection{Structure and molecular weight characterization of BCPs $1 \mathrm{a}-\mathrm{i}$}

The block composition ratios and absolute lengths of BCPs $\mathbf{1 a - i}$ were confirmed by ${ }^{1} \mathrm{H}$ NMR analysis: The block lengths of the polystyrene macro-initiators $\mathbf{3 a}-\mathbf{c}$ were determined by ${ }^{1} \mathrm{H}$ NMR end-group analysis using the trimethylsilyl (TMS) group on the initiator as an integration reference. ${ }^{29}$ The styrene:imidazolium-styrene block ratios for each BCP were determined by integrating and comparing distinct ${ }^{1} \mathrm{H}$ NMR signals indicative of each block. The ionic block lengths were then calculated based on the alkyl block lengths and the block composition ratios. These results were further confirmed by TMS end-group analysis ${ }^{29}$ and the copolymerization monomer-to-initiator ratios. Exposure of solutions of $\mathbf{1 a}-\mathbf{i}$ to $\mathrm{AgNO}_{3}$ did not show precipitate formation, indicating negligible $\mathrm{Br}^{-}$in the BCPs. Consequently, the absolute $M_{\mathrm{n}}$ values for BCPs 1a-i could be calculated by simply multiplying the absolute block lengths (as determined by ${ }^{1} \mathrm{H}$ NMR) by the MWs of the repeat units (see the Supplementary Data). 
Unfortunately, GPC and other conventional polymer MW determination methods (e.g., matrix-assisted laser desorption ionization time-of-flight mass spectrometry, and dynamic light scattering) could not be used to confirm the MW, PDI, or block structure of 1a-i because of the unusual solubility and other physical properties of these noncharged-charged BCPs, as previously reported. ${ }^{11 a}$ A modified GPC method that enables poly(IL) MW determination was recently reported; ${ }^{27 c}$ however, we could not modify the GPC system we used in such a fashion, and the hybrid characteristics of noncharged-charged BCPs still presented solubility difficulties. Instead, a combination of alternative methods (i.e., surfactant behavior and solubility analysis, diffusion-ordered spectroscopy (DOSY), small-angle X-ray scattering (SAXS) studies) was used to verify the block architectures of $\mathbf{1 a}-\mathbf{i}$ and to differentiate their behavior from that of a physical blend of polystyrene and $\operatorname{poly}(\mathbf{2 a})-\operatorname{poly}(\mathbf{2 c})$ homopolymers, as described previously ${ }^{11 \mathrm{a}}$ (see the Supplementary Data).

\subsection{Characterization of the morphologies formed by BCPs 1 a-i}

Table 1. Summary of the room-temperature morphologies formed by thermally annealed BCPs $\mathbf{1 a - i}$ according to SAXS analysis.

\begin{tabular}{|c|c|c|c|c|c|}
\hline BCP & $\mathbf{n}$ & $\mathbf{m}$ & $\mathbf{R}$ & Morphology & Observed q/q*100 values \\
\hline $\mathbf{1 a}$ & 25 & 25 & methyl & Hex (weakly ordered & $\sqrt{1},(\sqrt{3}), \sqrt{4}, \sqrt{7},(\sqrt{ } 9)$ \\
\hline $1 b$ & 25 & 25 & $n$-butyl & Hex (weakly ordered) & $\sqrt{ } 1,(\sqrt{ } 3), \sqrt{ } 4, \sqrt{ } 7,(\sqrt{ } 9)$ \\
\hline 1c & 25 & 25 & $n$-decyl & Disordered & (none) \\
\hline 1d & 20 & 30 & methyl & $\mathrm{S}_{\mathrm{BCC}}$ & $\sqrt{ } 2, \sqrt{ } 4, \sqrt{6}, \sqrt{ } 8$ \\
\hline 1e & 20 & 30 & $n$-butyl & $\mathrm{S}_{\mathrm{BCC}}$ & $\sqrt{ } 2, \sqrt{ } 4, \sqrt{ } 6, \sqrt{ } 8$ \\
\hline 1f & 20 & 30 & $n$-decyl & Disordered & (none) \\
\hline $1 g$ & 15 & 35 & methyl & $\mathrm{S}_{\text {LLP }}($ weakly ordered) & form factor scattering \\
\hline $1 \mathrm{~h}$ & 15 & 35 & $n$-butyl & $\mathrm{S}_{\text {LLP }}($ weakly ordered) & form factor scattering \\
\hline $\mathbf{1 i}$ & 15 & 35 & $n$-decyl & Disordered & (none) \\
\hline
\end{tabular}

The room-temperature $\left(25^{\circ} \mathrm{C}\right)$ morphological behavior of BCPs 1a-i (following meltphase annealing at $175^{\circ} \mathrm{C}$ for $30 \mathrm{~min}$ ) from SAXS analysis is summarized in Table 1 . The SAXS profiles of these samples at room-temperature after annealing are shown in Fig. 3 . The data confirm microphase separation for all BCP samples containing methyl or $n$-butyl substituents on the imidazolium ring (i.e., 1a, 1b, 1d, 1e, 1g, and $\mathbf{1 h}$ ), regardless of the styrene:imidazoliumstyrene block ratios tested. In contrast, the BCPs with the longer $n$-decyl substituent on the imidazolium block show no evidence of microphase separation. We speculate that by increasing the non-ionic character of the imidazolium substituent opposite the phenyl linker, the ability of the system to separate the charged units and create a thermodynamic advantage through microphase separation is becoming severely diminished. Differential scanning calorimetry (DSC) measurements revealed no evidence of any observable thermal transitions between -40 and $180{ }^{\circ} \mathrm{C}$, and thus provided no additional insight into the microphase-separated state of the systems beyond that provided by SAXS. That is, neither glass transitions for the polymer blocks nor melt transitions associated with the alkyl side-chains on the imidazolium units could be detected in these samples. Details describing the DSC measurements and representative DSC data can be found in the Supplementary Data. 


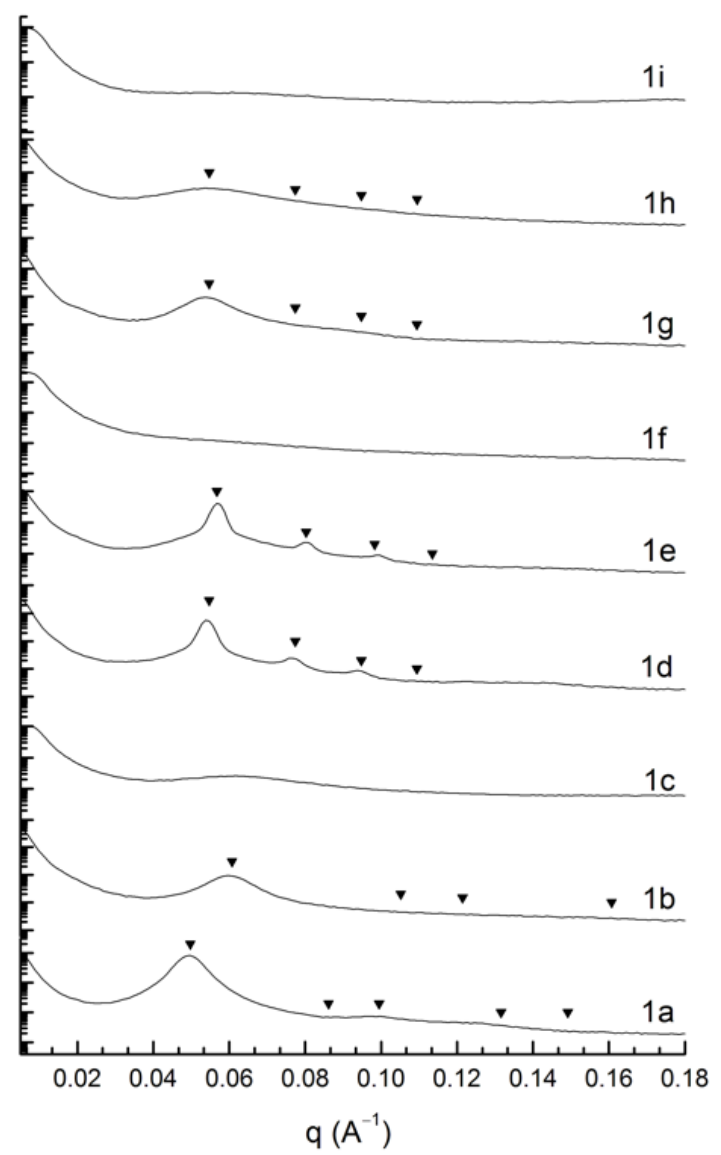

Fig. 3. SAXS profiles of BCPs $1 \mathrm{a}-\mathbf{i}$ at $25^{\circ} \mathrm{C}$ after annealing at $175^{\circ} \mathrm{C}$ for $30 \mathrm{~min}$. The inverted triangles indicate the expected SAXS reflection positions for spheres ordered in a BCC lattice $(\mathbf{1 d}, \mathbf{1 e}, \mathbf{1 g}, \mathbf{1 h})$ and hexagonally packed cylinders $(\mathbf{1 a}, \mathbf{1 b})$.

Of the microphase-separated methyl and $n$-butyl samples, those in which the styrene:imidazolium-styrene block ratios are fixed at 20:30 (1d, 1e) exhibit a particularly high degree of order. In fact, both the methyl and $n$-butyl BCPs at this composition produce clear diffraction peaks consistent with the symmetry of spheres on a body-centered-cubic ( $\mathrm{S}_{\mathrm{BCC}}$ ) lattice, with the expected $\mathrm{q} / \mathrm{q}^{*}$ ratios of $\sqrt{2}\left(\mathrm{q}_{110}\right), \sqrt{4}, \sqrt{6}, \sqrt{8}$, etc. Here, $\mathrm{q}^{*}=\mathrm{q}_{100}$ is absent due to the reflection conditions associated with the BCC symmetry. Importantly, the $\mathrm{q}_{110}$ interplanar spacings at $25^{\circ} \mathrm{C}$ imply cubic unit cell lattice constants of 16.6 and $15.5 \mathrm{~nm}$ for the methyl and $n$-butyl substituted samples, respectively. Such large lattice constants suggest chain stretching in these systems is significant, given the small degrees of polymerization (DPs) used in this initial study. Mahanthappa and coworkers saw similar evidence of chain stretching in analogous noncharged-charged BCPs prepared by postpolymerization modification; ${ }^{18}$ however, their studies were limited to compositions in which the charged imidazolium blocks comprised only the minority component (i.e., $\leq 17 \mathrm{~mol} \%$ of the total number of repeat units). This was largely a constraint of the limited DPs and MW control that they could achieve using a creative multi-step, postpolymerization modification strategy to produce the charged imidazolium block. In their case, they used NMP of styrene and 4-vinylbenzyl chloride to produce modifiable polymeric precursors that were then quaternized with various $N$-alkylimidazoles, followed by anion- 
exchange with $\mathrm{Tf}_{2} \mathrm{~N}^{-18}$ In contrast, our sequential ATRP of styrene and monomers $\mathbf{2 a}-\mathbf{c}$ reported here enables these imidazolium-containing BCPs to be produced more directly/easily and at compositions spanning the entire mole fraction spectrum.

It is significant that the sphere morphology is present in BCP $\mathbf{1}$ homologs in which the charged imidazolium block comprises just $60 \mathrm{~mol} \%$ of the repeat units in the chain. For BCPs in which the repeat units of the two blocks are of similar volumes, such compositions would sit at the edge of the lamellar phase boundary. This suggests the volume increase associated with adding the charged imidazolium substituent to the phenyl ring in the polystyrene backbone is substantial, and creates significant packing differences between the ionic and nonionic blocks in the system. Mahanthappa and coworkers actually saw lamellar morphologies forming at imidazolium-containing repeat unit compositions as low as $8 \mathrm{~mol} \%{ }^{18}$ Their results, taken together with ours, emphasize the rather extreme asymmetry in packing introduced when one block carries the charged imidazolium species.

Based on these observations, one would expect BCPs 1a (methyl) and 1b (n-butyl) with noncharged:charged block ratios fixed at 25:25 to exhibit phase behavior bounded by lamellae and sphere phases. However, the broadness evident in the scattering peaks (even after annealing at $175^{\circ} \mathrm{C}$ ) suggests that while these samples clearly phase-separate, they are challenged to find a well-ordered morphology at these compositions. In Fig. 3, inverted triangular markers above the SAXS profiles of BCPs $\mathbf{1 a}$ and $\mathbf{1 b}$ indicate the positions of expected reflections for cylinders on a hexagonal lattice (i.e., at $\mathrm{q} / \mathrm{q}^{*}$ ratios of $\sqrt{1}\left(\mathrm{q}_{100}\right), \sqrt{3}, \sqrt{ } 4, \sqrt{7}, \sqrt{9}$, etc.). At this point, a definitive assignment of the formed phase is not possible based on scattering data alone.

The most asymmetric BCP samples (1g and 1h) with styrene:imidazolium-styrene block ratios fixed at 15:35 are also only weakly ordered. Based on the propensity of samples $\mathbf{1 d}$ and 1e to adopt the $\mathrm{S}_{\mathrm{BCC}}$ lattice, the volume fraction of the styrene block in $\mathbf{1 g}$ and $\mathbf{1 h}$ may be significantly lower than the $0.10-0.14$ range associated with the $S_{\mathrm{BCC}}$ phase. Samples in which the volume fraction of one block approaches values less than $10 \%$ have been found in many instances to adopt a liquid-like packing of spherical domains $\left(S_{\text {LLP }}\right) .{ }^{11 \mathrm{~b}, 30}$ The scattering signature for these systems is entirely consistent with that observed for $\mathbf{1 g}$ and $\mathbf{1 h}$, with characteristic shoulder adjacent to a broad primary peak associated with form factor oscillations. Notably, these same scattering signatures were also characteristic of the $S_{\mathrm{BCC}}$-forming samples (1d, 1e) prior to annealing (c.f., Fig. 4). 


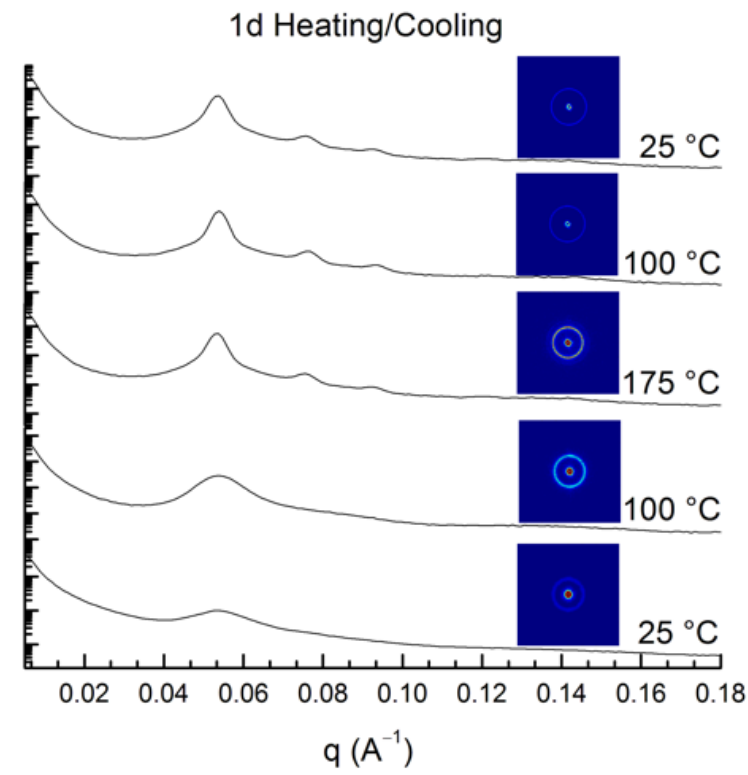

Fig. 4. SAXS profiles of BCP 1d throughout the thermal annealing procedure. Peak positions are consistent with those for spheres packed in a BCC lattice.

Inspection of each series of samples organized by fixed styrene:imidazolium-styrene block ratio reveals that an increase in the alkyl substituent chain length on the imidazolium ring produces a decrease in the principle domain spacing for the system. This seemingly counterintuitive result supports the argument that adding nonionic character (via choice of alkyl chain length) to the ionic block reduces the degree of segregation in the system, and helps relieve the high degree of chain stretching required to reach a free energy minimum. This shift towards decreased segregation levels supports the argument that the $n$-decyl substituent on the imidazolium ring is indeed acting to compatibilize the system. These data also suggest that a judicious choice of alkyl substituent on the imidazolium-styrene monomer provides a discrete method for tuning the Flory interaction parameter for such systems. If true, one might be able to control the ability of the system to adopt phases like bicontinuous gyroid, which have demonstrated a clear preference to form under more weakly segregated conditions. ${ }^{11 \mathrm{~b}, 18}$

Similar to other imidazolium-based noncharged-charged BCPs we produced in the past, ${ }^{11}$ many of the samples produced here required an initial heating step to achieve their highest level of order. For example, Fig. 4 shows that there is a sufficient delay in obtaining highly ordered $\mathrm{BCC}$ spheres in the first heating cycle. The gradual ordering process, evident by an evolution of the diffraction pattern from one with broad, form-factor oscillations at room temperature, to one with multiple, easily resolved diffraction peaks at $175{ }^{\circ} \mathrm{C}$ and above. As mentioned previously, this type of behavior in which liquid-like packing of spheres $\left(\mathrm{S}_{\mathrm{LLP}}\right)$ often exists prior to development of the fully organized $\mathrm{S}_{\mathrm{BCC}}$ lattice is fairly common in other BCP systems. ${ }^{11 \mathrm{~b}, 30} \mathrm{As}$ shown in Fig. 4, the highly ordered structure established during the initial heating remains preserved during subsequent cooling, with no evidence of any return to the initial, less ordered state. 


\section{Summary}

We have shown that imidazolium-based noncharged-charged diblock copolymers (1) capable of forming ordered, phase-separated nanostructures can be synthesized by the direct, sequential ATRP of styrene and styrenic imidazolium bis(trifluoromethyl)sulfonamide monomers containing different-length alkyl side-chains. To our knowledge, the synthesis of imidazolium-based BCPs of this type via direct ATRP of immiscible co-monomers is unprecedented. SAXS studies on thermally annealed initial examples of $\mathbf{1}$ with a total of 50 repeat units and styrene:imidazolium-styrene repeat unit ratios of 25:25, 20:30, and 15:35 showed that their ability to form ordered nanostructures in their neat states depends on both the block ratio and the length of the alkyl side-chain on the imidazolium monomer. It was found that only the BCP samples with the two shortest alkyl chains (methyl and $n$-butyl) on the imidazolium monomer form ordered nanostructures over the range of compositions tested: The methyl- and $n$-butyl-imidazolium homologs with the most symmetric noncharged:charged repeat unit ratio (25:25) exhibited scattering signatures for weakly-ordered cylinders (hexagonal lattice), while the methyl- and $n$-butyl-imidazolium BCPs with progressively more asymmetric block ratios (20:30 and 15:35) formed spheres in a body-centered cubic lattice or with more disordered liquid-like packing character. In contrast, none of the BCPs containing the longest imidazolium side-chain ( $n$-decyl) exhibited any ordered phase-separated structures regardless of block composition. This result suggests that overly long alkyl chains on the ionic imidazolium block can make it more miscible with the hydrophobic styrene block and less conducive to mutual phase separation. Collectively, these initial results with this directly polymerized ILbased BCP system show that it may be able to target the formation of specific phase-separated morphologies via judicious choice of styrene:imidazolium-styrene block ratio and the nature of the substituent(s) on the imidazolium-styrene monomer.

We are currently investigating if the homologs of $\mathbf{1}$ that exhibit ordered morphologies can be fabricated into supported membranes so that we can evaluate the contribution of their ordered nanostructures in $\mathrm{CO}_{2}$ /light gas separation applications. We are also in the process of exploring a wider range of block length ratios and the use of imidazolium-styrene monomers with other alkyl side-chains in order to systematically map out the phase behavior of this BCP system as a function of these two variables. Also of interest is examining the effect of different associated anions in the imidazolium block on BCP phase behavior. It has recently been reported that the counterion in noncharged-charged BCPs can play an important role in controlling their morphologies. ${ }^{31}$ One specific objective in these structure-morphology investigations is seeing if we can obtain a bicontinuous cubic/gyroid phase, which has so far been elusive in imidazoliumbased noncharged-charged BCPs. The ability to directly synthesize the target BCPs via sequential ATRP of styrene and a selected imidazolium-styrene monomer allows ready access to the desired test materials. Future studies will also focus on more detailed morphological analysis of the less ordered systems using complementary visualization techniques such as electron microscopy imaging.

\section{Experimental section}




\subsection{Materials and general procedures}

1-Bromodecane, sodium hydride, imidazole, 1-methylimidazole, 1-butylimidazole, 4vinylbenzyl chloride, 2-(trimethylsilyl)ethanol, $\alpha$-bromoisobutyryl bromide, triethylamine, copper(I) bromide, butyronitrile, Dowex 50Wx4 ion-exchange resin, benzoyl peroxide, $N, N, N^{\prime}, N^{\prime}, N^{\prime \prime}$-pentamethyldiethylenetriamine (PMDETA), and TEMPO were all purchased from the Sigma-Aldrich Co., and used as received. Styrene was purchased from the Sigma-Aldrich Co. and purified by passage over a column of basic alumina to remove the added radical inhibitor. Lithium bis(trifluoromethylsulfonyl)amide $\left(\operatorname{LiTf}_{2} \mathrm{~N}\right)$ was purchased as Fluorad ${ }^{\mathrm{TM}}$ Lithium Trifluoromethane Sulfonimide from the 3M Company. All solvents were purchased from Sigma-Aldrich or Mallinckrodt, Inc., and purified/dehydrated via $\mathrm{N}_{2}$-pressurized activated alumina columns, and de-gassed. The $\mathrm{H}_{2} \mathrm{O}$ used for synthesis was purified and deionized, with resistivity greater than $12 \mathrm{M} \Omega / \mathrm{cm}$. All polymerizations were carried out in a dry Ar atmosphere using standard Schlenk line techniques.

\subsection{Instrumentation}

${ }^{1} \mathrm{H}$ and ${ }^{13} \mathrm{C}$ NMR spectra were obtained using a Bruker 300 Ultrashield ${ }^{\mathrm{TM}}$ (300 MHz for $\left.{ }^{1} \mathrm{H}\right)$ spectrometer. Chemical shifts are reported in ppm relative to residual non-deuterated solvent. HRMS (ES) analysis was performed by the Central Analytical Facility in the Dept. of Chemistry and Biochemistry at the University of Colorado, Boulder. Gel permeation chromatography (GPC) was performed using a Viscotek GPC-Max chromatography system outfitted with three $7.5 \times 340 \mathrm{~mm}$ Polypore ${ }^{\mathrm{TM}}$ (Polymer Laboratories) columns in series, a Viscotek differential refractive index (RI) detector, and an Alltech column oven (mobile phase THF, $40{ }^{\circ} \mathrm{C}, 1 \mathrm{~mL} \mathrm{~min}{ }^{-}$ ${ }^{1}$ flow rate). Molecular weight data obtained on this GPC system were referenced to polystyrene molecular weight standards. NMR diffusion ordered spectroscopy (DOSY) experiments were performed using a Varian Inova-400NMR spectrometer. Small-angle X-ray scattering (SAXS) data were collected using a Rigaku SMax3000 High Brilliance three-pinhole SAXS system outfitted with a MicroMax-007HFM rotating anode $(\mathrm{Cu} \mathrm{K})_{\alpha}$, a Confocal Max-Flux Optic, a Gabriel-type multi-wire area detector, and a Linkam thermal stage. Differential scanning calorimetry (DSC) measurements were performed using a Mettler Toledo DSC823 ${ }^{\mathrm{e}}$ and a Julabo FT100 Intracooler. Energy-dispersive X-ray spectroscopy (EDS) was performed using a JEOL JSM-6480 scanning electron microscope with an elemental detection lower limit of carbon.

\subsection{Synthesis of 1-decylimidazole}

This compound was synthesized using a variation of the procedure previously reported. ${ }^{32}$ Sodium hydride (60\% dispersion in mineral oil, $1.56 \mathrm{~g}, 39.0 \mathrm{mmol}$ ) was suspended in dry THF $(50 \mathrm{~mL})$ under $\operatorname{Ar}$ at $0{ }^{\circ} \mathrm{C}$. Imidazole $(2.16 \mathrm{~g}, 31.7 \mathrm{mmol})$ was dissolved in dry THF $(20 \mathrm{~mL})$ and added drop-wise. After $30 \mathrm{~min}$, 1-bromodecane $(5.41 \mathrm{~g}, 24.5 \mathrm{mmol})$ was added. The resulting reaction mixture was then stirred at room temperature overnight. After careful addition of a few drops of water to quench the remaining sodium hydride, the organic solvent was removed in vacuo, and diethyl ether $(50 \mathrm{~mL})$ was added. This solution was then washed with water $(3 \times 50$ $\mathrm{mL}$ ), dried over anhydrous $\mathrm{MgSO}_{4}$, filtered, concentrated, and then purified by flash chromatography (ethyl acetate/hexanes $=10 / 1(\mathrm{v} / \mathrm{v})$ ) to give the product as a light yellow oil (yield: $4.38 \mathrm{~g}, 86 \%$ ). Spectroscopic and purity data matched those reported for this compound. ${ }^{32}$ 


\subsection{Synthesis of 1-(4-vinylbenzyl)-3-alkyl-imidazolium bis(trifluoromethylsulfonyl)amide monomers (2)}

These compounds were synthesized using a variation of the procedures previously reported. ${ }^{27,33}$ The methods below detail the synthesis of styrenic imidazolium monomer 2a-c.

\subsubsection{Synthesis of 1-(4-Vinylbenzyl)-3-methyl-imidazolium bis(trifluoromethylsulfonyl)amide (2a)}

(Note: This monomer has been reported in the literature, but the complete synthesis and characterization data for only its chloride derivative has been provided ${ }^{33}$ not the $\mathrm{Tf}_{2} \mathrm{~N}^{-}$analog 2a). 4-Vinylbenzyl chloride $(2.50 \mathrm{~g}, 16.4 \mathrm{mmol})$ and 1-methylimidazole $(1.48 \mathrm{~g}, 18.0 \mathrm{mmol})$ were stirred in acetonitrile $(100 \mathrm{~mL})$ at reflux for $18 \mathrm{~h}$. Upon cooling, the reaction mixture was concentrated to form a yellow viscous oil and washed with $\mathrm{Et}_{2} \mathrm{O}(3 \times 100 \mathrm{~mL})$. The resulting oil was then dissolved in $\mathrm{H}_{2} \mathrm{O}(100 \mathrm{~mL}), \operatorname{LiTf}_{2} \mathrm{~N}(5.17 \mathrm{~g}, 18.0 \mathrm{mmol})$ was added, and then the mixture stirred at room temperature for $12 \mathrm{~h}$. A yellow oil was then extracted from this aqueous mixture with $\mathrm{CH}_{2} \mathrm{Cl}_{2}(3 \times 50 \mathrm{~mL})$. The $\mathrm{CH}_{2} \mathrm{Cl}_{2}$ layer was then washed with $\mathrm{H}_{2} \mathrm{O}(3 \times 100 \mathrm{~mL})$, dried over anhydrous $\mathrm{MgSO}_{4}$, filtered, and concentrated to give monomer $\mathbf{2 a}$ as light yellow oil (yield: $6.83 \mathrm{~g}, 87 \%) .{ }^{1} \mathrm{H}$ NMR (300 MHz, $\left.\mathrm{CDCl}_{3}\right): \delta 8.77(\mathrm{~s}, 1 \mathrm{H}), 7.43(\mathrm{~d}, J=8.2 \mathrm{~Hz}, 2 \mathrm{H}), 7.32$ $(\mathrm{d}, J=8.2 \mathrm{~Hz}, 2 \mathrm{H}), 7.21(\mathrm{dt}, J=18.0,1.8 \mathrm{~Hz}, 2 \mathrm{H}), 6.69(\mathrm{dd}, J=17.6,10.9 \mathrm{~Hz}, 1 \mathrm{H}), 5.77(\mathrm{~d}, J=$ $17.6,1 \mathrm{H}), 5.31(\mathrm{~d}, J=10.9,1 \mathrm{H}), 5.28(\mathrm{~s}, 2 \mathrm{H}), 3.90(\mathrm{~s}, 3 \mathrm{H}) .{ }^{13} \mathrm{C}$ NMR $\left(75 \mathrm{MHz}, \mathrm{CDCl}_{3}\right)$ : 8139.06, 135.92, 135.80, 131.69, 129.24, 127.30, 123.96, 122.21, 122.01, 117.76, 115.64, 53.29, 36.39. IR (neat): $3153.53,1562.40,1347.39,1329.23,1178.28,1132.72,1051.69,994.24$, 920.00, 830.65, 789.12, 739.18, 715.92, 635.47. HRMS (ES) calcd. for $\mathrm{C}_{15} \mathrm{H}_{15} \mathrm{~F}_{6} \mathrm{~N}_{3} \mathrm{O}_{4} \mathrm{~S}_{2}\left(\mathrm{M}^{+}\right.$ $\mathrm{Tf}_{2} \mathrm{~N}^{-}$): 479.0408; observed: 479.0424. Since imidazolium-based ionic liquid compounds are known in the literature to have combustion issues for $\mathrm{C}, \mathrm{H}$, and $\mathrm{N}$ elemental analysis, ${ }^{34}$ the ${ }^{1} \mathrm{H}$ and ${ }^{13} \mathrm{C}$ NMR spectra for isolated 2a are provided in Supplementary Data to help confirm its purity.

\subsubsection{Synthesis of 1-(4-Vinylbenzyl)-3-butyl-imidazolium bis(trifluoromethylsulfonyl)amide $(2 b)^{27 a}$}

4-Vinylbenzyl chloride (2.00 g, $13.1 \mathrm{mmol})$ and 1-butylimidazole $(1.79 \mathrm{~g}, 14.4 \mathrm{mmol})$ were stirred in acetonitrile $(80 \mathrm{~mL})$ at reflux for $18 \mathrm{~h}$. Upon cooling, the reaction mixture was concentrated to form a yellow viscous oil and washed with $\mathrm{Et}_{2} \mathrm{O}(3 \times 80 \mathrm{~mL})$. The resulting oil was then dissolved in $\mathrm{H}_{2} \mathrm{O}(80 \mathrm{~mL}) . \quad \operatorname{LiTf}_{2} \mathrm{~N}(4.51 \mathrm{~g}, 15.7 \mathrm{mmol})$ was added, and then the mixture stirred at room temperature for $12 \mathrm{~h}$. A yellow oil was then extracted from this aqueous mixture with $\mathrm{CH}_{2} \mathrm{Cl}_{2}(3 \times 50 \mathrm{~mL})$. The $\mathrm{CH}_{2} \mathrm{Cl}_{2}$ layer was then washed with $\mathrm{H}_{2} \mathrm{O}(3 \times 100 \mathrm{~mL})$, dried over anhydrous $\mathrm{MgSO}_{4}$, filtered, and concentrated to give monomer $\mathbf{2 b}$ as light yellow oil (yield: $5.81 \mathrm{~g}, 85 \%$ ). Spectroscopic and purity data matched those reported for this compound. ${ }^{27 a}$

\subsubsection{Synthesis of 1-(4-Vinylbenzyl)-3-decyl-imidazolium bis(trifluoromethylsulfonyl)amide} (2c). 
4-Vinylbenzyl chloride (1.00 g, $6.55 \mathrm{mmol})$ and 1-decylimidazole (1.50 g, $7.20 \mathrm{mmol})$ were stirred in acetonitrile $(40 \mathrm{~mL})$ at reflux for $18 \mathrm{~h}$. Upon cooling, the reaction mixture was concentrated to form a yellow viscous oil and washed with $\mathrm{Et}_{2} \mathrm{O}(3 \mathrm{x} 40 \mathrm{~mL})$. The resulting oil was then dissolved in $\mathrm{H}_{2} \mathrm{O}(40 \mathrm{~mL}) . \operatorname{LiTf}_{2} \mathrm{~N}(2.26 \mathrm{~g}, 7.87 \mathrm{mmol})$ was added, and then the resulting mixture stirred at room temperature for $12 \mathrm{~h}$. A yellow oil was then extracted from this aqueous mixture with $\mathrm{CH}_{2} \mathrm{Cl}_{2}(3 \times 50 \mathrm{~mL})$. The $\mathrm{CH}_{2} \mathrm{Cl}_{2}$ layer was then washed with $\mathrm{H}_{2} \mathrm{O}(3 \mathrm{x}$ $100 \mathrm{~mL}$ ), dried over anhydrous $\mathrm{MgSO}_{4}$, filtered, and concentrated to give monomer 2c as light yellow oil (yield: $3.49 \mathrm{~g}, 88 \%)$. ${ }^{1} \mathrm{H}$ NMR $\left(300 \mathrm{MHz}, \mathrm{CDCl}_{3}\right)$ : $\delta 8.88(\mathrm{~s}, 1 \mathrm{H}), 7.44(\mathrm{~d}, J=8.2 \mathrm{~Hz}$, $2 \mathrm{H}), 7.33(\mathrm{~d}, J=8.2 \mathrm{~Hz}, 2 \mathrm{H}), 7.24(\mathrm{dt}, J=12.0,2.0 \mathrm{~Hz}, 2 \mathrm{H}), 6.69(\mathrm{dd}, J=17.6,10.9 \mathrm{~Hz}, 1 \mathrm{H})$, $5.78(\mathrm{~d}, J=17.6,1 \mathrm{H}), 5.31(\mathrm{~d}, J=10.8,1 \mathrm{H}), 5.31(\mathrm{~s}, 2 \mathrm{H}), 4.16(\mathrm{t}, J=7.4 \mathrm{~Hz}, 2 \mathrm{H}), 1.93-1.76$ $(\mathrm{m}, 2 \mathrm{H}), 1.39-1.17(\mathrm{~m}, 14 \mathrm{H}), 0.87(\mathrm{t}, J=7.0 \mathrm{~Hz}, 3 \mathrm{H}) .{ }^{13} \mathrm{C} \mathrm{NMR}\left(75 \mathrm{MHz}, \mathrm{CDCl}_{3}\right): \delta 139.19$, $135.83,135.58,131.69,129.29,127.41,122.45,122.24,122.08,117.82,115.76,53.46,50.49$, 31.95, 30.18, 29.51, 29.40, 29.34, 28.96, 26.24, 22.77, 14.21. IR (neat): 3146.88, 2927.24, 2856.85, 1560.77, 1456.43, 1348.21, 1182.03, 1133.87, 1053.89, 990.02, 914.86, 830.80, 788.70, 739.55, 653.29. HRMS (ES) calcd. for $\mathrm{C}_{24} \mathrm{H}_{33} \mathrm{~F}_{6} \mathrm{~N}_{3} \mathrm{O}_{4} \mathrm{~S}_{2}\left(\mathrm{M}^{+} \mathrm{Tf}_{2} \mathrm{~N}^{-}\right)$: 605.1817; observed: 605.2435. Since imidazolium-based ionic liquid compounds are known in the literature to have combustion issues for $\mathrm{C}, \mathrm{H}$, and $\mathrm{N}$ elemental analysis, ${ }^{34}$ the ${ }^{1} \mathrm{H}$ and ${ }^{13} \mathrm{C}$ NMR spectra for isolated $\mathbf{2 c}$ are provided in Supplementary Data to help confirm its purity.

\subsection{Synthesis of 2-(Trimethylsilyl)ethyl 2-bromo-2-methylpropanoate (TMS-EBMP).}

This compound was synthesized using the previously reported procedure. ${ }^{27 \mathrm{c}}$ Spectroscopic and purity data matched those reported for this compound. ${ }^{27 \mathrm{c}}$

\subsection{Synthesis of polystyrene macro-initiators $3 a-c$ via ATRP of styrene.}

These compounds were synthesized using a variation of the procedures previously reported. ${ }^{35}$ In a typical procedure, the desired amount of purified styrene and PMDETA were added to a flame-dried Schlenk flask and degassed by three freeze-pump-thaw cycles. After the flask was then allowed to warm to room temperature and back-filled with Ar, the desired amount of $\mathrm{CuBr}$ was added. The resulting mixture was then stirred at room temperature for $30 \mathrm{~min}$, and TMS-EBMP was added. The flask was then placed in a $90{ }^{\circ} \mathrm{C}$ oil bath for $22 \mathrm{~h}$. The contents of the flask were cooled to room temperature, dissolved in acetone, stirred with Dowex 50Wx4 ionexchange resin for $30 \mathrm{~min}$, and filtered through a short plug of basic alumina. The resulting solution was then concentrated in vacuo, diluted with $\mathrm{Et}_{2} \mathrm{O}$, precipitated by adding into $\mathrm{MeOH}$, and the precipitate recovered by filtration. This dissolution/precipitation procedure was repeated two more times to give the desired polystyrene macro-initiator $\mathbf{3}$ as a white solid (Table 1). ${ }^{1} \mathrm{H}$ NMR analysis of $\mathbf{3 a}-\mathbf{c}$ confirmed the absence of unreacted styrene monomer. The synthesis of

$\mathbf{3 a}$ is shown below as a representative example. The synthesis details for $\mathbf{3 b}$ and $\mathbf{3 c}$ are provided in the Supplementary Data. The DP and $M_{\mathrm{n}}$ values of $\mathbf{3 a}-\mathbf{c}$ were calculated based on the ${ }^{1} \mathrm{H}$ NMR end-group analysis (see the Supplementary Data).

\subsubsection{Example: synthesis of polystyrene macro-initiator $3 a$.}

Styrene $(2.50 \mathrm{~g}, 24.0 \mathrm{mmol})$ and PMDETA $(22.3 \mathrm{mg}, 0.129 \mathrm{mmol})$ were added to a flame-dried Schlenk flask and degassed by three freeze-pump-thaw cycles. The flask was then 
allowed to warm to room temperature, back filled with argon, and $\mathrm{CuBr}(18.4 \mathrm{mg}, 0.128 \mathrm{mmol})$ was added. The resulting mixture was stirred at room temperature for 30 minutes and TMSEBMP (229 mg, $0.857 \mathrm{mmol}$ ) was added. The flask was then place in an oil bath at $90{ }^{\circ} \mathrm{C}$ for 22 $\mathrm{h}$. The content of the flask was purified as described in general procedures to give $\mathbf{3 a}$ as white solid (yield: $2.25 \mathrm{~g}, 82 \%$ ). $\mathrm{DP}=25 ; \mathrm{PDI}=1.12 ; M_{\mathrm{n}}=2,871 \mathrm{~g} / \mathrm{mol}$ (calculated using ${ }^{1} \mathrm{H} \mathrm{NMR}$ polymer end-group analysis. See the Supplementary Data for details on how the DP and absolute $M_{\mathrm{n}}$ were determined using ${ }^{1} \mathrm{H} \mathrm{NMR}$ analysis).

\subsection{Synthesis of BCPs la-i via ATRP of imidazolium-styrene monomers $2 a-c$ from the polystyrene macro-initiators $3 a-c$.}

In a typical procedure, the appropriate amount of the desired imidazolium monomer $\mathbf{2}$, PMDETA, and butyronitrile were added to a flame-dried Schlenk flask and degassed by three freeze-pump-thaw cycles. After the flask was allowed to warm to room temperature and backfilled with $\mathrm{Ar}$, the appropriate amount of $\mathrm{CuBr}$ was then added. The resulting mixture was then stirred at room temperature for $30 \mathrm{~min}$, and then the appropriate amount of the desired polystyrene macro-initiator 3 was added. The flask was then placed in a $90{ }^{\circ} \mathrm{C}$ oil bath. Upon complete consumption of monomer 2 (as verified by ${ }^{1} \mathrm{H}$ NMR analysis), the contents of the flask were cooled to room temperature, diluted with acetone, stirred with Dowex 50Wx4 ion-exchange resin for $15 \mathrm{~min}$, and then filtered through a short plug of basic alumina. The resulting solution was then concentrated in vacuo, diluted with acetone, and added to a $\mathrm{MeOH} / \mathrm{H}_{2} \mathrm{O}(3 / 1$ (v/v)) mixture. The resulting precipitate was then dissolved in acetone, re-precipitated by adding into hexanes, and isolated by filtration. This dissolution/precipitation procedure was repeated one more time to give the desired BCP 1 as a white solid (Table 2). Contact of a solution of the final BCPs with $\mathrm{AgNO}_{3}$ showed no $\mathrm{AgBr}$ precipitate formation, indicating negligible $\mathrm{Br}^{-}$anion in the polymers. ${ }^{1} \mathrm{H}$ NMR analysis of the BCPs $1 \mathbf{1 a}-\mathbf{i}$ confirmed the absence of any residual unreacted monomer. The synthesis of $\mathbf{1 a}$ is shown below as a representative example. The synthesis details for BCPs $\mathbf{1 b}-\mathbf{i}$ are provided in the Supplementary Data. The block compositions and $M_{\mathrm{n}}$ values of $\mathbf{1 a}-\mathbf{i}$ were calculated based on the ${ }^{1} \mathrm{H}$ NMR analysis (see the Supplementary Data).

\subsubsection{Example: synthesis of BCP 1a.}

Imidazolium monomer 2a (418 mg, $0.871 \mathrm{mmol})$, PMDETA (24.0 mg, $0.139 \mathrm{mmol})$, and butyronitrile $(1 \mathrm{~mL})$ were added to a flame-dried Schlenk flask and degassed by three freezepump-thaw cycles. After the flask was allowed to warm to room temperature and back-filled with $\mathrm{Ar}, \mathrm{CuBr}(20.0 \mathrm{mg}, 0.139 \mathrm{mmol})$ was added. The resulting mixture was stirred at room temperature for $30 \mathrm{~min}$, and macro-initiator $3 \mathbf{a}(100 \mathrm{mg}, 0.0348 \mathrm{mmol})$ was added. The flask was then placed in a $90{ }^{\circ} \mathrm{C}$ oil bath and stirred. Upon complete consumption of monomer $\mathbf{2 a}$ (as verified by ${ }^{1} \mathrm{H}$ NMR analysis), the resulting reaction mixture was purified as described in the general procedure above to give BCP 1a as a white solid (yield: $0.303 \mathrm{~g}, 58 \%$ ). Block repeat unit molar ratio $=1: 1$ (styrene:imidazolium-styrene); block length composition $=25-b-25$ (styrene- $b$-imidazolium-styrene); $M_{\mathrm{n}}=14,856 \mathrm{~g} / \mathrm{mol}$ (calculated based on ${ }^{1} \mathrm{H}$ NMR analysis. See the Supplementary Data for details on how the copolymer block composition, block lengths, and $M_{\mathrm{n}}$ were determined). 


\subsection{General procedure for the verification of the controlled polymerization character for the ATRP of styrenic imidazolium monomers $2 a-c$.}

The appropriate amount of the desired styrenic imidazolium monomer 2, PMDETA, and butyronitrile were added to a flame-dried Schlenk flask and degassed by three freeze-pump-thaw cycles. After the flask was allowed to warm to room temperature and back-filled with Ar, the appropriate amount of $\mathrm{CuBr}$ was added. The resulting mixture was stirred at room temperature for $30 \mathrm{~min}$, and then the appropriate amount of TMS-EBMP or the desired polystyrene macroinitiator 3 was added. The flask was then placed in a $90{ }^{\circ} \mathrm{C}$ oil bath and stirred. At selected timed intervals, samples of the reaction mixture were taken, and the polymers were precipitated in $\mathrm{MeOH} / \mathrm{H}_{2} \mathrm{O}(3 / 1 \mathrm{v} / \mathrm{v})$ for NMR analysis (see the Supplementary Data for details).

\subsection{Small-Angle X-ray Scattering (SAXS).}

Small-angle X-ray scattering (SAXS) data were collected using a Rigaku S-Max 3000 High Brilliance three-pinhole SAXS system outfitted with a MicroMax-007HFM rotating anode $\left(\mathrm{Cu} \mathrm{K} \mathrm{K}_{a}, \lambda=1.54 \AA\right.$ ) , sample-to-detector distance of $2.19 \mathrm{~m}$, Confocal Max-Flux Optics, Gabriel multiwire area detector $(1024 \times 1024$ pixel resolution $)$, and a Linkam thermal stage. Copolymer samples were sandwiched between Kapton windows $(0.05 \mathrm{~mm}$ thick $\times 10 \mathrm{~mm}$ diameter). Before collection of temperature-dependent SAXS data, the sample stage temperature was allowed to equilibrate for 5 min under vacuum, unless otherwise stated. Data were collected under vacuum ( $\sim 100 \mathrm{mtorr})$ with exposure times of $1200 \mathrm{~s}$ for all samples. SAXS data were azimuthally integrated from the $2 \mathrm{D}$ detector patterns and plotted as logarithmic intensity vs. the scattering wave vector, $\mathrm{q}$, defined as $\mathrm{q}=(4 \pi / \lambda) \sin \left(2 \theta_{\mathrm{B}} / 2\right)$, where $2 \theta_{\mathrm{B}}$ is the angle between the incident and scattered waves.

\section{Acknowledgments}

Financial support for the work performed at CU Boulder was provided by the Department of Energy ARPA-e program (grant: DE-AR0000098) and matching funds from Total, S.A. (France). Financial support for the work performed at CSU was provided by the National Science Foundation (grant: CBET-1160026) and the ACS Petroleum Research Fund (grant: 52018-ND7). SAXS studies were performed at CSU using a regional instrument supported by the National Science Foundation MRI Program (grant: DMR-0821799).

\section{Appendix A. Supplementary data}

Supplementary data related to this article can be found at

\section{References}

(1) For recent reviews on poly(IL)s, see: (a) Green; O.; Grubjesic, S.; Lee, S.; Firestone, M. A. J. Macromol. Sci. C: Polym. Rev. 2009, 49, 339. (b) Yuan, J.; Mecerreyes, D.; Antonietti, M. Prog. Polym. Sci. 2013, 38, 1009. (c) Lu, J.; Yan, F.; Texter, J. Prog. Polym. Sci. 2009, 
34, 431. (d) Anderson, E.; Long, T. Polymer 2010, 51, 2447. (e) Jaeger, W.; Bohrisch, J.; Laschewsky, A. Prog. Polym. Sci. 2010, 35, 511.

(2) For a recent review on IL and IL monomer design, see: Green. M. D.; Long, T. E. $J$. Macromol. Sci. C: Polym. Rev. 2009, 49, 291.

(3) (a) Ding, S.; Tang, H.; Radosz, M.; Shen, Y. J. Polym. Sci. A: Polym. Chem. 2004, 42, 5794. (b) Bara, J. E.; Lessmann, S.; Gabriel, C. J.; Hatakeyama, E. S.; Noble, R. D.; Gin, D. L. Ind. Eng. Chem. Res. 2007, 46, 5397.

(4) (a) Ohno, H., Ito, K. Chem. Lett. 1998, 27, 751. (b) Ohno, H., Yoshizawa, M., Ogihara, W. Electrochim. Acta 2004, 50, 255. (c) Vygodskii, Y.; Shaplov, A.; Lozinskaya, E.; Lyssenko, K.; Golovanov, D.; Malyshkina, I.; Gavrilova, N.; Buchmeiser, M. Macromol. Chem. Phys. 2008, 209, 40.

(5) (a) Marcilla, R.; Curri, M. L.; Cozzoli, P. D.; Mart'inez, M. T.; Loinaz, I.; Grande, H.; Pomposo, J. A.; Mecerreyes, D. Small 2006, 2, 507. (b) Fukushima, T.; Kosaka, A.; Yamamoto, Y.; Aimiya, T.; Notazawa, S.; Takigawa, T.; Inabe, T.; Aida, T. Small 2006, 2, 554.

(6) (a) Ho, H. A.; Leclerc, M. J. Am. Chem. Soc. 2003, 125, 4412. (b) Ricks-Laskoski, H. L.; Snow, A. W. J. Am. Chem. Soc. 2006, 128, 12402.

(7) For a recent review on morphologies of BCPs, see: Mai, Y.; Eisenberg, A. Chem. Soc. Rev. 2012, 41, 5969.

(8) Vijayakrishna, K.; Jewrajka, S. K.; Ruiz, A.; Marcilla, R.; Pomposo, J. A.; Mecerreyes, D.; Taton, D.; Gnanou, Y. Macromolecules 2008, 41, 6299.

(9) Mori, H.; Yahagi, M.; Endo, T. Macromolecules 2009, 42, 8082.

(10) Karjalainen, E.; Chenna, N.; Laurinm"aki, P.; Butcher, S. J.; Tenhu, H. Polym. Chem. 2013, $4,1014$.

(11) (a) Wiesenauer, E. F.; Edwards, J. P.; Scalfani, V. F.; Bailey, T. S.; Gin, D. L. Macromolecules 2011, 44, 5075. (b) Scalfani, V. F.; Wiesenauer, E. F.; Ekblad, J. R.; Edwards, J. P.; Gin, D. L.; Bailey, T. S. Macromolecules 2012, 45, 4262. (c) Nguyen, P. T.; Wiesenauer, E. F.; Gin, D. L.; Noble, R. D. J. Membr. Sci. 2013, 430, 312. (d) Wiesenauer, E. F.; Nguyen, P. T.; Newell, B. S.; Bailey, T. S.; Noble, R. D.; Gin, D. L. Soft Matter 2013, 9, 7923.

(12) He, H.; Luebke, D.; Nulwala, H.; Matyjaszewski, K. Macromolecules 2014, 47, 6601. (DOI: 10.1021/ma501487u; Publication Date (Web): September 17, 2014.)

(13) Texter, J.; Vasantha, V. A.; Crombez, R.; Maniglia, R.; Slater, L.; Mourey, T. Macromol. Rapid Commun. 2012, 33, 69.

(14) Agudelo, N. A.; Elsen, A. M.; He, H.; López, B. L.; Matyjaszewski, K. J. Polym. Sci. A: Polym. Chem. 2014, published on-line (DOI: 10.1002/pola.27300).

(15) Chanthad, C.; Masser, K. A.; Xu, K.; Runt, J.; Wang, Q. J. Mater. Chem. 2012, $22,341$.

(16) Li, J.; Liang, J.; Wu, W.; Zhang, S.; Zhang, K.; Zhou, H. New J. Chem. 2014, 38, 2508.

(17) (a) Stancik, C. M.; Lavoie, A. R.; Schutz, J.; Achurra, P. A.; Lindner, P.; Gast, A. P.; Waymouth, R. M. Langmuir 2004, 20, 596. (b) Stancik, C. M.; Lavoie, A. R.; Achurra, P. A.; Waymouth, R. M.; Gast, A. P. Langmuir 2004, 20, 8975.

(18) Weber, R. L.; Ye, Y.; Schmitt, A. L.; Banik, S. M.; Elabd, Y. A.; Mahanthappa, M. K. Macromolecules 2011, 44, 5727.

(19) Green, M. D.; Choi, J.-H.; Winey, K. I.; Long, T. E. Macromolecules 2012, 45, 4749.

(20) Gu, Y.; Timothy P. Lodge, T. P. Macromolecules 2011, 44, 1732. 
(21) (a) Rao, A. H. N.; Thankamony, R. L.; Kim, H.-J.; Namb, S.; Kim, T.-H. Polymer 2013, 54, 111.(b)Rao, A. H. N.; Kim, H.-J.; Namb, S.; Kim, T.-H. Polymer 2013, 54. 6918. (c) Rao, A. H. N.; Kim, H.-J.; Namb, S.; Kim, T.-H. Int. J. Hydrogen Energy 2014, 39, 5919.

(22) Han, G. L.; Xu, P. Y.; Zhou, K.; Zhang, Q. G.; Zhu, A. M.; Liu. Q. L. J. Membr. Sci. 2014, 464,72 .

(23) (a) Green, M. D.; Wan, D.; Hemp, S. T.; Choi, J.-H.; Winey, K. I.; Heflin, J. R.; Long, T. E. Polymer 2012, 53, 3677. (b) Jangu, C.; Wang, J.-H. H.; Wang, D.; Sharick, S.; Heflin, J. R.; Winey, K. I.; Colby, R. H.; Long, T. E. Macromol. Chem. Phys. 2014, 215, 1319.

(24) Adzima, B. J.; Venna, S. R.; Klara, S. S.; He, H.; Zhong, M.; Luebke, D. R.; Mauter, M. S.; Matyjaszewski, K.; Nulwala, H. B. J. Mater. Chem. A 2014, 2, 7967.

(25) Gao, R.; Wang, D.; Heflin, J. R.; Long, T. E. J. Mater. Chem. 2012, 22, 13473.

(26) Bara, J. E.; Lessmann, S.; Gabriel, C. J.; Hatakeyama, E. S.; Noble, R. D.; Gin, D. L. Ind. Eng. Chem.Res. 2007, 46, 5397.

(27) (a) Tang, J.; Tang, H.; Sun, W.; Radosz, M.; Shen, Y. J. Polym. Sci. A: Polym. Chem. 2005, 43, 5477. (b) He, X.; Yang, W.; Pei, X. Macromolecules 2008, 41, 4615. (c) He, H.; Zhong, M.; Adzima, B.; Luebke, D.; Nulwala, H.; Matyjaszewski, K. J. Am. Chem. Soc. 2013, 135, 4227. (d) Oh, J. Y.; Chi, W. S.; Lee, J. H.; Kim, J. H.; Kang, S. W. Ind. Eng. Chem. Res. 2013, 52, 9607. (e) Chi, W. S.; Hong, S. U.; Jung, B.; Kang. S. W.; Kang, Y. S.; Kim, J. H. J. Membr. Sci. 2013, 443, 54. (f) Karjalainen, E.; Izquierdo, D. F.; MartiCentelles, V.; Luis, S. V.; Tenhu, H.; Garcia-Verdugo. E. Polym. Chem. 2014, 5, 1437. (g) Hu, H.; Yuan, W.; Zhao, H.; Baker, G. L. J. Polym. Sci. A: Polym. Chem. 2014, 52, 121. (h) He, H.; Averick, S.; Roth, E.; Luebke, D.; Nulwala, H.; Matyjaszewski, K. Polymer 2014, 55, 3330. (i) He, H.; Zhong, M.; Luebke, D.; Nulwala, H.; Matyjaszewski, K. J. Polym. Sci. A: Polym. Chem. 2014, 52, 2175.

(28) Long, S.; Wan, F.; Yang, W.; Guo, H.; He, X.; Ren, J.; Gao, J. J. Appl. Polym. Sci. 2013, 2687.

(29) Päch, M.; Zehm, D.; Lange, M.; Dambowsky, I.; Weiss, J.; Laschewsky, A. J. Am. Chem. Soc. 2010, 132, 8757.

(30) (a) Guo, C.; Bailey, T. S. Soft Matter 2010, 6, 4807. (b) Scalfani, V. F.; Bailey, T. S. Macromolecules 2011, 44, 6557.

(31) Sing, C. E.; Zwanikken, J. W.; Olvera de la Cruz, M. Nat. Mater. 2014, 13, 694.

(32) Zhao, H.; Foss, F. W. Jr.; Breslow, R. J. Am. Chem. Soc. 2013, 130, 12590.

(33) Zhao, D.; Fei, Z.; Ang, W. H.; Dyson, P. J. Small 2006, 2, 879.

(34) Schenkel, M. R.; Zhao, R.; Robertson, L. A.; Wiesenauer, B. R.; Clark, N. A.; Gin, D. L. Liq. Cryst. 2013, 40, 1067, and references therein.

(35) Xia, J.; Matyjaszewski, K. Macromolecules 2011, 30, 7697. 


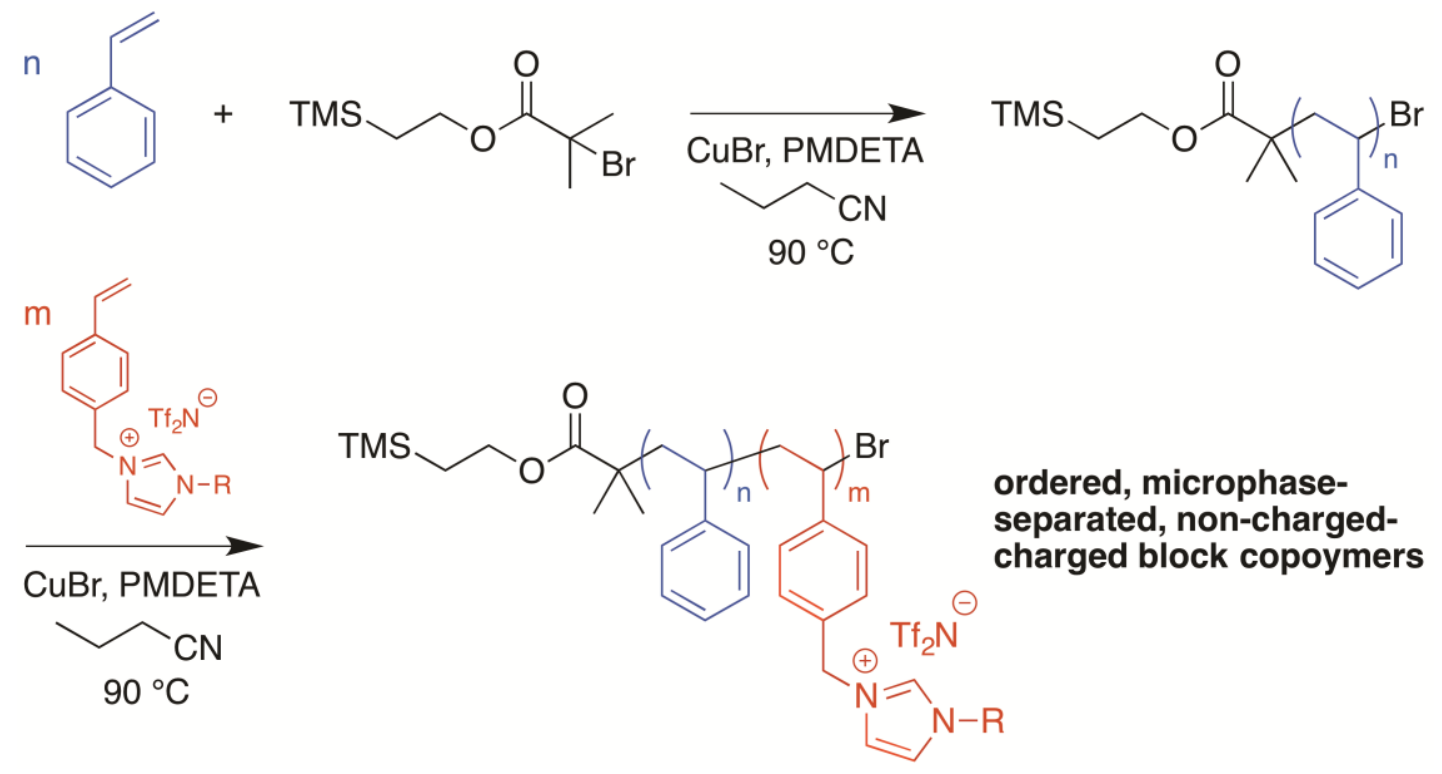

